\title{
On soft topological categories
}

\author{
Gülay Oğuz*(D), M. Habil Gürsoy (iD, İlhan İçen \\ Department of Mathematics, Faculty of Arts and Sciences, Inönü University, Malatya, Turkey
}

\begin{abstract}
In this paper, we introduce the notion of a soft topological category as a natural consequence of the existence of topological category and soft category. Some examples of the soft topological categories are given. The properties of soft topological category are investigated and some important results are obtained. Also, the notion of topological functor is extended to the notion of soft topological functor. Finally, we present some examples about it.
\end{abstract}

Mathematics Subject Classification (2010). 18A05, 22A05, 55U40, 97H40

Keywords. soft set, soft topological group, category, functor, soft category, soft topological category

\section{Introduction}

The basis of mathematical science is the logical system. However, it is not always possible to obtain accurate and complete knowledge for complex problems in physics, chemistry, engineering and many other fields. For centuries, many scientists, including mathematicians, have searched for uncertain and incomplete information pushing the boundaries of classical logic. As a result of these investigations, many theories have been developed. One of these theories is the soft set theory introduced by Molodtsov in 1999 [8].

Soft set theory is an important mathematical approach modeling the uncertainty that comes from overcoming some of the difficulties that exist in traditional theories. This theory has created a wide field of study in different fields such as game theory, probability and measurement theory in a short time. Especially mathematicians have studied this theory with their algebraic and topological aspects $[1-3,5,7,9,12,13]$. After Molodtsov described the soft set theory, Maji and et.al. proposed different operators on soft sets and examined some of their fundamental properties [6]. The first topological studies on this theory were presented by Shabir and Naz [12]. They studied the separation axioms on a soft topological space by defining soft topological space. Later, Cagman and et.al., Aygunoglu and Aygun, and Min obtained some basic results by studying on soft topological spaces and algebraic structures $[3,5,7]$.

Beside of these, the soft set theory has been also studied from viewpoint of category theory which is defined as a classification of mathematical structures [4]. We refer to $[10,11,14]$ for these studies. Sardar and Gupta defined the concept of soft category and

\footnotetext{
*Corresponding Author.

Email addresses: gulay.oguz@inonu.edu.tr (G. Oğuz), mhgursoy@gmail.com (M.H. Gürsoy), ilhan.icen@inonu.edu.tr (İ. İçen)

Received: 19.10.2017; Accepted: 12.06.2018
} 
introduced the basic concepts of soft category theory [11]. Moreover, they compared soft category with fuzzy category.

In this work, we introduce a soft approach to the topological categories by taking Sardar and Gupta's work one step further. More precisely, we present the concept of soft topological category which is a new concept obtained by combining topological category and soft set theory. We also give some characterizations about the soft topological category. Some important concepts of topological category are applied to soft set theory and some new concepts such as soft topological subcategory and soft topological functor are presented. After that, their some properties are discussed. It can be summarized that this paper categorically extends the boundaries of the soft set theory which is in the process of rapid development and presents a new viewpoint in the topological sense.

\section{Preliminaries}

In this section, we will first recall basic definitions and some properties of soft set theory, category theory and topological category to provide the integrity of this article. For more details, we refer to $[2,4,8,9]$.

Let $U$ be an initial universe set and $A$ be a non-emty set of parameters.

Definition 2.1. [8] A pair $(F, A)$ is called a soft set over $U$, where $F$ is a mapping defined by

$$
F: A \longrightarrow P(U)
$$

It can be said that a soft set over $U$ is actually a parametrized family of subsets of the universe $U$.

Definition 2.2. [6] Let $(F, A)$ and $(G, B)$ be two soft sets over a universe $U$. $(F, A)$ is said to be a soft subset of $(G, B)$ if

i. $A \subset B$, and

ii. $\forall a \in A, F(a)$ and $G(a)$ are identical approximations.

We denote it by $(F, A) \widetilde{C}(G, B)$.

Definition 2.3. [6] A soft set $(F, A)$ over $U$ is called a null soft set denoted by $\Phi$, if $\forall a \in A, F(a)=\emptyset$.

Definition 2.4. [6] A soft set $(F, A)$ over $U$ is said to be an absolute soft set denoted by $\tilde{A}$, if $\forall a \in A, F(a)=U$.

Definition 2.5. [13] Let $\tau$ be a topology defined on a group $G$. Let $(F, A)$ be a non-null soft set defined over $G$. Then, the triplet $(F, A, \tau)$ is called a soft topological group over $G$ if

i. $F(a)$ is a subgroup of $G$ for all $a \in A$

ii. the mapping $(x, y) \longmapsto x-y$ of the topological space $F(a) \times F(a)$ onto $F(a)$ is continuous for all $a \in A$.

Definition 2.6. [13] Let $(F, A, \tau)$ be a soft topological group over $G$. Then $(F, A, \tau)$ is said to be soft trivial if $F(a)=\{0\}$ for all $a \in A$ and is said to be soft whole if $F(a)=G$ for all $a \in A$, where 0 denotes the identity of $G$.

Definition 2.7. [13] Let $(F, A, \tau)$ be a soft topological group over $G$. Then $(K, B, \tau)$ is said to be a soft topological (resp. normal subgroup) subgroup of $(F, A, \tau)$ if

i. $B \subset A$,

ii. $K(b)$ is a subgroup (resp. normal subgroup) of $F(b)$ for all $b \in \operatorname{supp}(K, B)$,

iii. the mapping $(x, y) \longrightarrow x-y$ of the topological space $K(b) \times K(b)$ onto $K(b)$ is continuous for all $b \in \operatorname{supp}(K, B)$. 
Definition 2.8. [13] Let $(F, A, \tau)$ and $(K, B, \tau)$ be two soft topological groups over $G$ and $G^{\prime}$, where $\tau$ and $\tau^{\prime}$ are topologies defined over $G$ and $G^{\prime}$, respectively. Let $f: G \longrightarrow G^{\prime}$ and $g: A \longrightarrow B$ be two mappings. Then the pair $(f, g)$ is called a soft topological group homomorphism if the following conditions are satisfied:

i. $f$ is a group epimorphism and $g$ is surjection.

ii. $f(F(a))=K(g(a))$.

iii. $f_{a}:\left(F(a), \tau_{F(a)}\right) \longrightarrow\left(K(g(a)), \tau_{K(g(a))}^{\prime}\right)$ is continuous.

Then $(F, A, \tau)$ is said to be soft topologically homomorphic to $\left(K, B, \tau^{\prime}\right)$ and denoted by $(F, A, \tau) \sim\left(K, B \tau^{\prime}\right)$.

Here, we introduce the concept of soft topological ring because we need it in the future.

Definition 2.9. [13] Let $\tau$ be a topology defined on a commutative ring $R$. Let $(F, A)$ be a non-null soft set defined over $R$. Then, the triplet $(F, A, \tau)$ is called soft topological ring over $R$ if for all $a \in A$,

i. $F(a)$ is a subring of $G$ for all $a \in A$

ii. the mapping $F(a) \times F(a) \longrightarrow F(a)$ defined by $(x, y) \longmapsto x-y$ is continuous.

iii. the mapping $F(a) \times F(a) \longrightarrow F(a)$ defined by $(x, y) \longmapsto x \cdot y$ is continuous.

Definition 2.10. [13] Let $(F, A, \tau)$ and $(G, B, \tau)$ be two soft topological rings over $R$ and $R^{\prime}$, where $\tau$ and $\tau^{\prime}$ are topologies defined over $R$ and $R^{\prime}$, respectively. Let $f: R \longrightarrow R^{\prime}$ and $g: A \longrightarrow B$ be two mappings. Then, the pair $(f, g)$ is called a soft topological ring homomorphism if the following conditions are satisfied:

i. $f$ is a ring epimorphism and $g$ is surjection.

ii. $f(F(a))=G(g(a))$.

iii. $f_{a}:\left(F(a), \tau_{F(a)}\right) \longrightarrow\left(G(g(a)), \tau_{G(g(a))}^{\prime}\right)$ is continuous.

Then $(F, A, \tau)$ is said to be soft topologically homomorphic to $\left(G, B, \tau^{\prime}\right)$ and, denoted by $(F, A, \tau) \sim\left(G, B \tau^{\prime}\right)$.

The concepts of category and topological category are important in the category theory. We define these concepts here:

Definition 2.11. [4] A category $\mathcal{C}$ is a structure $\mathcal{C}=(\operatorname{Mor}(\mathcal{C}), O b(\mathcal{C}), \alpha, \beta, m, i)$ given by a set $\operatorname{Mor}(\mathrm{C})$ of arrows, a set $O b(\mathrm{C})$ of objects and four structure maps;

$$
\operatorname{Mor}(\mathrm{C}) \times \operatorname{Mor}(\mathrm{C}) \stackrel{m}{\longrightarrow} \operatorname{Mor}(\mathrm{C}) \stackrel{\alpha, \beta}{\longrightarrow} \operatorname{Ob}(\mathrm{C}) \stackrel{i}{\longrightarrow} \operatorname{Mor}(\mathrm{C})
$$

The maps $\alpha$ and $\beta$ give for each arrow $g \in \operatorname{Mor}(\mathcal{C})$ its source $\alpha(g)$ and its target $\beta(g)$.

The map $m$ is defined for any pair of arrows $f, g$ with $\alpha(f)=\beta(g)$, and assigns to this pair the composition $(f, g)$ also denoted $f \circ g$.

The map $i$, called inclusion map, assigns to each object $x \in X$ the identity arrow at $x$, denoted $i(x)$.

Definition 2.12. [9] A topological category $\mathcal{C}=(\operatorname{Mor}(\mathcal{C}), O b(\mathcal{C}), \alpha, \beta, m, i)$ is a category such that $\operatorname{Mor}(\mathrm{C})$ and $O b(\mathrm{C})$ are sets equipped with a topology and the four structure maps are continuous with respect to these two topologies.

Definition 2.13. [11] Let $\mathcal{C}$ be a category, let $\mathcal{P}(\mathcal{C})$ be set of all subcategories of $\mathcal{C}$, and let $A$ be set of parameters. A pair $(F, A)$ is called a soft category over $\mathcal{C}$, where $F$ is a mapping given by

$$
F: A \longrightarrow \mathcal{P}(\mathcal{C})
$$

and $F(x)$ is a subcategory of $\mathcal{C}$.

In general, a soft category over $\mathcal{C}$ is said to be a parametrized family of subcategories of the category $\mathcal{C}$. The simplest example of a soft category is given as follows: 
Example 2.14. Let $(F, A)$ be a soft set over $U$. Then the set $U$ can be considered as a category $\mathcal{C}$ by defining $O b(\mathcal{C})=U, \mathcal{C}(X, X)=I_{X}$ and otherwise $\mathcal{C}(X, Y)=\emptyset$ for all $X, Y \in O b(\mathrm{C})$. Since $(F, A)$ is a soft set over $U, F(a)$ is a subset of $U$ for all $a \in A$. It is clear that if we consider $F(a)$ with its identity arrow, it becomes a subcategory of the category $\mathcal{C}$. Therefore, we say that $(F, A)$ is a soft category over $\mathfrak{C}$.

In this manner, every soft set can be considered as a soft category over the universe $U$.

\section{Soft topological categories}

Definition 3.1. Let $\mathcal{C}$ be a category whose object and arrow sets are topological spaces. Let $F: A \longrightarrow P(\mathcal{C})$ be a mapping, where $P(\mathcal{C})$ is the set of all subcategories of $\mathcal{C}$, and $A$ is the set of parameters. If the following conditions are satisfied, the pair $(F, A)$ is called a soft topological category over $\mathcal{C}$

i. $F(a)$ is a subcategory of $\mathcal{C}$ for all $a \in A$

ii. The structure maps of the subcategory $F(a)$ are continuous with respect to the topologies induced by $O b(\mathcal{C})$ and $\operatorname{Mor}(\mathcal{C})$ for all $a \in A$.

Note that if $\mathcal{C}$ is a topological category, it is sufficient that only the first condition of the above definition is satisfied in order to the pair $(F, A)$ to be defined as a soft topological category. In other words, the soft topological category $(F, A)$ can be considered as a parameterized family of subcategories of the topological category $\mathcal{C}$.

Example 3.2. Let $\mathcal{C}$ denotes the category of topological groups. Let us consider the set $A=\{$ normal, finite, abelian $\}$ of the paramaters. $F(a)$ is normal, finite and abelian topological subgroups for all $a \in A$, respectively. These subgroups are topological subcategories of $\mathcal{C}$. Since the conditions of Definition 3.1 are satisfied, the pair $(F, A)$ is a soft topological category on $\mathcal{C}$.

Example 3.3. Let $(F, A)$ be a soft topological group over the group $G$ with a topology $\tau$. Indeed, since $(F, A)$ is a soft topological group over the group $G, F(a)$ is a topological subgroup of $G$ for all $a \in A$. Also, since every group can be considered as a category with only one object, $F(a)$ is a topological subcategory of $G$. Therefore, $(F, A)$ is a soft topological category over $G$.

Likewise, it is straighforward to see that

Example 3.4. A soft topological ring $(F, A)$ over the $\operatorname{ring} R$ with identity can be considered as a soft topological category over the category $R$ because each ring is considered as a category with only one object.

Theorem 3.5. Every soft category on a topological category is a soft topological category.

Proof. Let $\mathcal{C}$ be a topological category and let $(F, A)$ be a soft category over $\mathcal{C}$. Then $F(a)$ is a subcategory of $\mathcal{C}$ for all $a \in A$. Thus, $F(a)$ is a topological subcategory of $\mathcal{C}$ with recpect to the topologies induced by $\operatorname{Mor}(\mathcal{C})$ and $O b(\mathcal{C})$ for all $a \in A$. Hence, $(F, A)$ is also a soft topological category over $\mathrm{C}$.

Remark 3.6. Each soft category $\mathcal{C}$ can be transformed into a soft topological category by equipping both $\mathrm{Ob}(\mathrm{C})$ and $\operatorname{Mor}(\mathrm{C})$ with discrete or indiscrete topology. But every soft category over a category is not a soft topological category.

Definition 3.7. Let $(F, A)$ be a soft topological category over the category $\mathcal{C}$. A pair $(H, B)$ is called a soft topological subcategory of $(F, A)$ if

i. $B \subset A$.

ii. $H(a)$ is a topological subcategory of $F(a)$ for all $a \in B$.

Example 3.8. Let $(F, A)$ be the soft topological category given in Example 3.2. Let us consider another soft topological category $(H, B)$ over the same topological group, where 
$B=\{$ abelian $\}$ and $H$ (abelian) is the subcategory of all finite abelian groups. Obviously, the pair $(H, B)$ is obtained as a soft topological subcategory of $(F, A)$.

Theorem 3.9. If $(H, B)$ is a soft topological subcategory of $(F, A)$ and $(K, C)$ is a soft topological subcategory of $(H, B)$, then $(K, C)$ is the soft topological subcategory of $(F, A)$.

Proof. The proof follows immediately from Definition 3.7.

Definition 3.10. Let both $(F, A)$ and $(H, B)$ be soft topological categories on the category C. These soft topological categories are called soft topological equal if $(F, A)$ is a soft topological subcategory of $(H, B)$ and $(H, B)$ is a soft topological subcategory of $(F, A)$.

Definition 3.11. Let $(H, B)$ be soft topological subcategory of $(F, A)$ over $\mathcal{C}$.

i. If $H(a)$ is a full subcategory of $F(a)$ for all $a \in B$, then $(H, B)$ is called a full soft topological subcategory of $(F, A)$.

ii. If $H(a)$ is a wide subcategory of $F(a)$ for all $a \in B$, then $(H, B)$ is called a wide soft topological subcategory of $(F, A)$.

Example 3.12. The soft topological subcategory $(H, B)$ given in Example 3.8 is a full soft topological subcategory of $(F, A)$.

Definition 3.13. Let $(F, A)$ and $(H, B)$ be soft topological categories over $\mathcal{C}$ and $\mathcal{D}$, respectively. Let $g: A \longrightarrow B$ be a mapping and $\mathfrak{K}: \mathcal{C} \longrightarrow \mathcal{D}$ be a functor. Then the pair $(\mathfrak{K}, g)$ is called a soft topological functor if the following conditions are satisfied:

i. $\mathfrak{K}$ is full and $g$ is surjection.

ii. $\mathfrak{K}(F(a))=H(g(a))$ for all $a \in A$.

ii. $\mathfrak{K}_{a}: F(a) \longrightarrow H(g(a))$ is continuous for all $a \in A$.

From the definition, it follows that a soft topological functor $(\mathfrak{K}, g)$ is a mapping of soft topological categories. Thus, we obtain a new category whose objects are soft topological categories and whose arrows are soft topological functors.

Example 3.14. Let $(H, B)$ be a soft topological subcategory of $(F, A)$. Together with the inclusion map $i: B \longrightarrow A$ and the identity functor $\mathcal{J}: \mathcal{C} \longrightarrow \mathrm{C}$, the pair $(\mathcal{J}, i)$ is a soft topological functor from $(H, B)$ to $(F, A)$.

Example 3.15. Let $(F, A)$ and $(H, B)$ be soft topological groups on groups $G$ and $G^{\prime}$, respectively. Also, let the pair $(f, g)$ be a soft topological group homomorphism from $(F, A)$ to $(H, B)$. We know from Example 3.3 that the soft topological groups $(F, A)$ and $(H, B)$ are soft topological categories over $G$ and $G^{\prime}$, respectively. Thus, the pair $(f, g)$ is a soft topological functor.

Example 3.16. As in the above example, let $(F, A)$ and $(H, B)$ be soft topological rings over $R$ and $R^{\prime}$ rings, respectively. We know from Example 3.4 that the soft topological rings $(F, A)$ and $(H, B)$ are soft topological categories over $R$ and $R^{\prime}$, respectively. If the pair $(f, g)$ is a soft topological ring homomorphism from $(F, A)$ to $(H, B)$, then $(f, g)$ is obtained as a soft topological functor between the soft topological categories $(F, A)$ and $(H, B)$.

Definition 3.17. Let $(F, A)$ and $(H, B)$ be soft topological categories over $\mathcal{C}$. Take Cop $^{\text {op }}$ as the dual category of $\mathcal{C}$. Then $(F, A)^{o p}=\left(F^{o p}, A\right)$, defined by $F^{o p}(a)=(F(a))^{o p}$ for all $a \in A$, is called the dual soft topological category of $(F, A)$. It is obvious that $(F, A)^{o p}$ is a soft topological category over $\mathcal{C}^{o p}$.

The following proposition follows from the corresponding result of dual category in the category theory.

Proposition 3.18. $\left((F, A)^{o p}\right)^{o p}=(F, A)^{o p}$ 
Here, we examine the soft topological versions of concepts such as initial, terminal and zero object in category theory.

Definition 3.19. Let $(F, A)$ be a soft topological category over $\mathcal{C}$. If $F(a)$ has initial object as a category for all $a \in A$, then $(F, A)$ is called a soft topological category with initial objects over $\mathcal{C}$.

It is clear that if $\mathcal{C}$ is a category with an initial object, then $(F, A)$ needs not have the initial objects.

Definition 3.20. Let $(F, A)$ be a soft topological category over $\mathcal{C}$. If $F(a)$ has terminal object as a category for all $a \in A$, then $(F, A)$ is called a soft topological category with terminal objects over $\mathcal{C}$.

It is clear that if $\mathcal{C}$ is a category with an terminal object, then $(F, A)$ needs not have the terminal objects.

Proposition 3.21. If $(F, A)$ is a soft topological category with initial (terminal) objects over $\mathcal{C}$, then $(F, A)^{o p}$ is a soft topological category with terminal (initial) objects over $\mathcal{C}$

Proof. By the Definition 3.19 (resp. Definition 3.20), $F(a)$ has initial (resp.terminal) object over $\mathcal{C}$ for all $a \in A$. In category theory, as is well known, every initial (resp. terminal) object of a category becomes terminal (resp. initial) object in its dual category. So $F(a)^{o p}$ has terminal (resp. initial) object over $\mathcal{C}$. This completes the proof.

Definition 3.22. Let $(F, A)$ be a soft topological category over $\mathcal{C}$. If $F(a)$ has zero object as a category for all $a \in A$, then $(F, A)$ is called a soft topological category with zero objects over $\mathcal{C}$.

Proposition 3.23. If $(F, A)$ is a soft topological category over $\mathcal{C}$, then any two initial (resp. terminal) objects in $(F, A)$ are isomorphic.

Proof. Let $(F, A)$ be a soft topological category over $\mathcal{C}$. Suppose that $(F, A)$ has two initial (resp. terminal) objects. Then $F(a)$ has two initial (resp. terminal) objects, as category for all $a \in A$. We know from category theory that any two initial (resp. terminal) objects are isomorphic. Therefore, the proof is completed.

From the above proposition, we can explicitly write the following result.

Corollary 3.24. The objects of the same type in a soft topological category are isomorphic.

Definition 3.25. Let $(F, A)$ be a soft topological category over $\mathcal{C}$. Then $(F, A)$ is called transitive if $F(a)$ is transitive as a category for all $a \in A$.

Remark 3.26. From soft category theory, it is well known that a soft category has a zero object if and only if it is transitive. The following theorem is a topological analoque of this statement.

Corollary 3.27. Let $(F, A)$ be a soft topological category with an initial and a terminal object over $\mathcal{C}$. Then $(F, A)$ has a zero object if and only if $(F, A)$ is transitive.

\section{References}

[1] U. Acar, F. Koyuncu and B. Tanay, Soft sets and soft rings, Comput. Math. Appl. 59, 3458-3463, 2010.

[2] H. Aktas and N. Cagman, Soft sets and soft groups, Inform. Sci. 77 (13), 2726-2735, 2007.

[3] A. Aygunoglu and A. Aygun, Some notes on soft topological spaces, Neural Comput. Appl. 22 (1), 113-119, 2012. 
[4] R. Brown and T. Porter, Category Theory: an abstract setting for analogy and comparison, in: What is Category Theory? Advanced Studies in Mathematics and Logic, Polimetrica Publisher, Italy, 257-274, 2006.

[5] N. Cagman, S. Karatas and S. Enginoglu, Soft topology, Comput. Math. Appl. 62 (1), 351-358, 2011.

[6] P.K. Maji, R. Biswas and A.R. Roy, Soft set theory, Comput. Math. Appl. 45 (4-5), 555-562, 2003.

[7] W.K. Min, A Note on Soft Topological Spaces, Comput. Math. Appl. 62, 3524-3528, 2011.

[8] D.A. Molodtsov, Soft set theory-First results, Comput. Math. Appl. 37 (4-5), 19-31, 1999.

[9] H. Nurusev, Topological grupoids, M.Sc. Thesis, Gazi University, Ankara, Turkey, 1995.

[10] S. Oztunc, Some properties of soft categories, Int. J. Model. Optim. 6 (2),2016.

[11] S.K. Sardar and S. Gupta, Soft category theory-an introduction, J. Hyperstruct. 2, 118-135, 2013.

[12] M. Shabir and M. Naz, On soft topological spaces, Comput. Math. Appl. 61 (7), 1786-1799, 2011.

[13] T. Shah and S. Shaheen, Soft topological groups and rings, Ann. Fuzzy Math. Inform. 7 (5), 725-743, 2014.

[14] O. Zahiri, Category of soft sets, An. Univ. Craiova Ser. Mat. Comput. Sci. 40 (2), 154-166, 2013. 